\title{
Sporadic SICs and the Normed Division Algebras
}

\author{
Blake C. Stacey ${ }^{1}$ \\ ${ }^{1}$ Department of Physics, University of Massachusetts Boston, \\ 100 Morrissey Blvd., Boston, MA 02125, United States
}

(Dated: September 4, 2018)

\begin{abstract}
Recently, Zhu classified all the SIC-POVMs whose symmetry groups act doubly transitively. Lattices of integers in the complex numbers, the quaternions and the octonions yield the key parts of these symmetry groups.
\end{abstract}

The problem of the SIC-POVMs [1-3] has a classic feel: It is easy to state, it continues to prove fiendishly hard to solve, and it makes unforeseen connections between disparate subjects. A symmetric, informationally complete, positive-operator valued measure - a SIC-POVM, or just a SIC-is a set of $d^{2}$ vectors $\left|\psi_{j}\right\rangle$ in a $d$-dimensional Hilbert space such that

$$
\left|\left\langle\psi_{j} \mid \psi_{k}\right\rangle\right|^{2}=\frac{d \delta_{j k}+1}{d+1} .
$$

In practice, it is often convenient to consider the equivalent set of rank-1 projectors, $\Pi_{j}=\left|\psi_{j}\right\rangle\left\langle\psi_{j}\right|$. Either way, such a set defines a quantum measurement operation with interesting properties [4-9]. The question is whether SICs exist for all values of $d$. The growing list of both exact and high-precision numerical solutions [7, 8] is encouraging, but as yet, we have no proof one way or the other.

A SIC is group covariant if it can be constructed by starting with a single vector (the fiducial) and acting upon that vector with the elements of some group. All known SICs are group covariant, although since group covariance simplifies the search process, this could be a matter of the light being under the lamppost. Furthermore, in all cases but one, that group is a WeylHeisenberg group. Working in dimension $d$, let $\omega_{d}=$ $e^{2 \pi i / d}$, and define the shift and phase operators

$$
X|j\rangle=|j+1\rangle, Z|j\rangle=\omega_{d}^{j}|j\rangle,
$$

where the shift is modulo $d$. Products of powers of $X$ and $Z$, together with dimension-dependent phase factors that we can neglect for the present purposes, define the Weyl-Heisenberg group.

In $d=2$, we can draw a SIC in the Bloch representation. Any qubit SIC forms a tetrahedron inscribed in the Bloch sphere [2]. Two SICs in higher dimensions will be important for our purposes. First is the Hesse SIC in $d=3$, constructed by applying the Weyl-Heisenberg group to the fiducial

$$
\left|\psi_{0}^{(\text {Hesse })}\right\rangle=\frac{1}{\sqrt{2}}(0,1,-1)^{\mathrm{T}} .
$$

Second is the Hoggar $S I C$ in $d=8$. We have multiple choices of fiducial in this case, but they all yield structures that are equivalent up to unitary or antiunitary transformations, so for brevity we speak of "the" Hoggar SIC [10]. One viable fiducial [11] is

$$
\left|\psi_{0}^{(\text {Hoggar })}\right\rangle \propto(-1+2 i, 1,1,1,1,1,1,1)^{\mathrm{T}} .
$$

The Hoggar SIC is the only known case where the group that constructs the SIC from the fiducial is not the WeylHeisenberg group for $d$ dimensions itself [10]. Instead, we use the tensor product of three copies of the qubit WeylHeisenberg group.

The SICs in dimensions 2 and 3, as well as the Hoggar SIC in dimension 8, stand apart in some respects from the other known solutions [8, 12]. They lie outside the algebraic number theory framework of Appleby et al. [8]. Either their dimensions are too small, or (in the case of the Hoggar SIC) they have the wrong symmetry group. We can think of them as the sporadic SICs. This list encompasses all of the SICs whose symmetry groups act doubly transitively on their projectors: the qubit SICs, the Hesse SIC in dimension 3, and the Hoggar SIC [13]. (The other SICs in $d=3$ do not have doubly-transitive symmetry groups, but should still be counted as sporadic [8].) I will now relate the doubly-transitive SICs with the complex numbers $\mathbb{C}$, the quaternions $\mathbb{H}$ and the octonions $\mathbb{0}$.

The Eisenstein integers [14] are complex numbers of the form

$$
z=a+b \omega, \text { where } \omega=e^{2 \pi i / 3} \text { and } a, b \in \mathbb{Z} .
$$

In the complex plane, they form a hexagonal lattice, designated $A_{2}$. A unit among integers is an integer whose multiplicative inverse is also an integer. Within the familiar set $\mathbb{Z}$, we have only two: namely, +1 and -1 . However, in the Eisenstein integers, there exist more choices. The group of units in the ring of Eisenstein integers is

$$
\left\{ \pm 1, \pm \omega, \pm \omega^{2}\right\} \text {. }
$$

Note the presence of the geometrical operations that take an equilateral triangle to itself: We see the identity, rotation by $1 / 3$ of a circle, and rotation by $2 / 3$ of a circle. So, modulo some signs, we have the symmetry operations that rotate a regular tetrahedron around the axis of one vertex, holding that vertex fixed.

A qubit SIC is a tetrahedron inscribed in the Bloch sphere, and unitary operations on qubit state space are rotations of that sphere. Therefore, the unitaries which 
hold one vertex of a SIC fixed and permute the other three form a group that is isomorphic to $\mathbb{Z}_{3}$.

In other words, the stabilizer group for each projector in a tetrahedral SIC is the unit group of the Eisenstein integers, quotiented by a small simple group.

Moving to the next normed division algebra, what about the quaternions $\mathbb{H}$ ? We can define a set of integers for $\mathbb{H}$, the so-called Hurwitz integers [14]. These are the quaternions whose coefficients are either all integers in $\mathbb{Z}$ or all half-integers. The units of the Hurwitz integers form the "binary tetrahedral group" [14, 15]. Thought of geometrically, the 24 units of the Hurwitz integers are the vertices of a polytope, the 24-cell, and they are the root vectors of the $D_{4}$ lattice. The binary tetrahedral group is known to be isomorphic to the matrix group $S L(2,3)$.

And $S L(2,3)$ is isomorphic to the stabilizer group for each of the projectors in the Hesse SIC. Therefore, we can say that the stabilizer of any element in the Hesse SIC is given by the unit group of the Hurwitz integers.

The octonions $\mathbb{O}$ also have integers among them [15, 16]. The construction relevant for our purposes is known as the Cayley integers. These form a lattice, specifically, the $E_{8}$ lattice scaled by a factor $1 / \sqrt{2}$. Exactly 240 elements in the Cayley integers have unit norm; they correspond to the root vectors of the $E_{8}$ lattice. Do they form a group of units, as we saw in $\mathbb{C}$ and in $\mathbb{H}$ ? Not exactly, because the octonions are not associative.
However, we can still avail ourselves of a group structure. To do so, we define an automorphism of the octonions as an invertible linear map from $\mathbb{O}$ to $\mathbb{O}$ that preserves the multiplication structure. The automorphism group of the integral octonions has order 12096 [15], and is sometimes written $G_{2}(\mathbb{Z})$. The structure of $G_{2}(\mathbb{Z})$ has been worked out, and is given by

$$
G_{2}(\mathbb{Z}) \cong P S U(3,3) \rtimes \mathbb{Z}_{2} .
$$

Zhu [13] identified $P S U(3,3)$ as isomorphic to the stabilizer of each projector in the Hoggar SIC.

In summary, then: For each of the normed division algebras $\mathbb{C}, \mathbb{H}$ and $\mathbb{O}$, we can build a set of integers whose symmetries are, up to factors of $\mathbb{Z}_{2}$, the stabilizer groups for projectors in the tetrahedral SICs, the Hesse SIC and the Hoggar SIC. (The factors of $\mathbb{Z}_{2}$ in dimensions 2 and 8 can be accounted for by considering the pair of a fiducial with its counterpart in a twinned SIC, related to the original SIC by an antiunitary operation [11]. Note that Eq. (3) is invariant under complex conjugation, but Eq. (4) is not.) Recall that we noticed the appearance of the $A_{2}, D_{4}$ and $E_{8}$ lattices. So, we can say that the doubly transitive SICs fall into an ADE classification [17]. The occurrence of these lattices, moreover, connects the SIC question to the problem of sphere packing [18], another topic in which the solutions in one dimension can be vexingly unrelated to solutions for others.
[1] G. Zauner, Quantum Designs - Foundations of a Noncommutative Theory of Designs. PhD thesis, University of Vienna (1999). http://www.gerhardzauner.at/ qdmye.html.

[2] J. M. Renes, R. Blume-Kohout, A. J. Scott and C. M. Caves, "Symmetric informationally complete quantum measurements," Journal of Mathematical Physics 45, 6 (2004), 2171, arXiv:quant-ph/0310075.

[3] A. J. Scott and M. Grassl, "SIC-POVMs: A new computer study," Journal of Mathematical Physics 51, 4 (2010), 042203, arXiv:0910.5784 [quant-ph].

[4] C. A. Fuchs, "QBism: The Perimeter of Quantum Bayesianism," arXiv:1003.5209 [quant-ph] (2010).

[5] C. A. Fuchs and R. Schack, "Quantum-Bayesian Coherence," Reviews of Modern Physics 85, 4 (2013), 16931715, arXiv:1301.3274 [quant-ph].

[6] C. A. Fuchs and B. C. Stacey, "Some negative remarks on operational approaches to quantum theory." In Quantum Theory: Informational Foundations and Foils (Springer, 2016). arXiv:1401.7254 [quant-ph].

[7] M. A. Graydon and D. M. Appleby, "Quantum conical designs," Journal of Physics A 49, 8 (2016), 085301, arXiv:1507.05323 [quant-ph].

[8] D. M. Appleby, S. Flammia, G. McConnell and J. Yard, "Generating ray class fields of real quadratic fields via complex equiangular lines," arXiv:1604.06098 [math.NT] (2016).

[9] H. Zhu, "Quasiprobability representations of quantum mechanics with minimal negativity," arXiv:1604.06974 [quant-ph] (2016).
[10] H. Zhu, Quantum State Estimation and Symmetric Informationally Complete POMs. PhD thesis, National University of Singapore (2012).

http://scholarbank.nus.edu.sg/bitstream/handle/ 10635/35247/ZhuHJthesis.pdf.

[11] A. Szymusiak and W. Słomczyński, "Informational power of the Hoggar SIC-POVM," arXiv:1512.01735 [quant-ph] (2015).

[12] B. C. Stacey, "SIC-POVMs and Compatibility among Quantum States," arXiv:1404.3774 [quant-ph] (2014).

[13] H. Zhu, "Super-symmetric informationally complete measurements," Annals of Physics 362 (2015), 311-26, arXiv:1412.1099 [quant-ph].

[14] J. C. Baez, "On Quaternions and Octonions: Their Geometry, Arithmetic, and Symmetry by John H. Conway and Derek A. Smith," Bulletin of the American Mathematical Society 42 (2005), 229-43. http://math.ucr. edu/home/baez/octonions/conway_smith/.

[15] R. A. Wilson, The Finite Simple Groups (Springer, 2009).

[16] J. C. Baez, G. Egan and T. Silverman, "Integral Octonions," http://math.ucr.edu/home/baez/octonions/ integers/ (2016).

[17] U. Schreiber, "ADE Classification," https://ncatlab. org/nlab/show/ADE+classification (2015).

[18] M. Viazovska, "The sphere packing problem in dimension 8," arXiv:1603.04246 [math.NT] (2016). 